\title{
Older multimorbid patients' experiences on integration of services: a systematic review
}

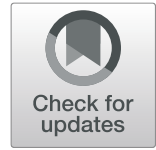

Lilian Keene Boye ${ }^{1,2,3^{*}}$ D, Christian Backer Mogensen ${ }^{1,2,3}$, Tine Mechlenborg ${ }^{4,5}$, Frans Boch Waldorff and

Pernille Tanggaard Andersen ${ }^{7}$

\begin{abstract}
Background: Half of the older persons in high-income counties are affected with multimorbidity and the prevalence increases with older age. To cope with both the complexity of multimorbidity and the ageing population health care systems needs to adapt to the aging population and improve the coordination of long-term services. The objectives of this review were to synthezise how older people with multimorbidity experiences integrations of health care services and to identify barriers towards continuity of care when multimorbid.

Methods: A systematic literature search was conducted in February 2018 by in Scopus, Embase, Cinahl, and Medline using the PRISMA guidelines. Inclusion criteria: studies exploring patients' point of view, $\geq 65$ and multimorbid. Quality assessment was conducted using COREQ. Thematic synthesis was done.

Results: Two thousand thirty studies were identified, with 75 studies eligible for full text, resulting in 9 included articles, of generally accepted quality.

Integration of health care services was successful when the patients felt listened to on all the aspects of being individuals with multimorbidity and when they obtained help from a care coordinator to prioritize their appointments. However, they felt frustrated when they did not have easy access to their health providers, when they were not listened to, and when they felt they were discharged too early. These frustrations were also identified as barriers to continuity of care.

Conclusions: Health care systems needs to adapt to people with multimorbidity and find solutions on ways to create flexible systems that are able to help older patients with multimorbidity, meet their individual needs and their desire to be involved in decisions regarding their care. A Care coordinator may be a solution.
\end{abstract}

Keywords: Older patients', Multimorbidity, Experiences, Integration of services, Continuity of care

\section{Background}

The population of those aged 65 and above is increasing, and life expectancy has increased by 30 years worldwide in the twentieth century $[1,2]$. With increased age, individuals commonly have two or more chronic conditions, often referred to as multimorbidity $[1,3,4]$. The prevalence of multimorbidity rises with age [5], and nearly 62 and $82 \%$ of those aged 65 years and older and aged more than 85 years, respectively, are individuals with multimorbidity [4]. Patients with multimorbidity have a high

\footnotetext{
* Correspondence: Lilian.keene.boye@rsyd.dk

${ }^{1}$ University Hospital of Southern Jutland, Kresten Philipsens vej 15, indgang F, 6200 Aabenraa, Denmark

${ }^{2}$ Focused Research Unit of Emergency Medicine, Department of Regional Health Research, University of Southern Denmark, Odense, Denmark Full list of author information is available at the end of the article
}

risk of a reduced quality of life, functional decline, and increased utilization of health care services [4]. Further, their pathway through health care systems can be difficult [6]. They may also need complex care, and they have very specific health care needs [3]. Consequently, health care systems worldwide find it challenging to provide care for these patients $[4,7]$, which emphasizes the importance of well-coordinated care [8], successful communication, and high interpersonal skills that, when combined, provide a high level of "continuity of care" [9-11]. "Continuity of care" is defined as how patients' experiences care as coherent and linked over time [11].

Continuity of care is important but may be difficult to establish for people with multimorbidity through the variety of organizations and places involved [9].

(c) The Author(s). 2019 Open Access This article is distributed under the terms of the Creative Commons Attribution 4.0 International License (http://creativecommons.org/licenses/by/4.0/), which permits unrestricted use, distribution, and reproduction in any medium, provided you give appropriate credit to the original author(s) and the source, provide a link to the Creative Commons license, and indicate if changes were made. The Creative Commons Public Domain Dedication waiver (http://creativecommons.org/publicdomain/zero/1.0/) applies to the data made available in this article, unless otherwise stated. 
Continuity of care as a concept is often presumed rather than defined, and the term is perceived differently [9-12]. Other concepts, for example, "coordination of care", "continuum of care", or "integrated care" are commonly used to mean "continuity of care" [11-14]. Put simply, "continuity of care" refers to the way in which patients experience both integration of services and coordination between providers and occurs when elements of care are connected and maintained over time [11]. Nonetheless, "continuity of care" also regards the manner in which patients experience transitions, their relationship with their health care providers and relatives, the transition of information, and consistency of care and personnel [11].

To adapt to both the complexity of multimorbidity and the aging population, health care systems need to improve the coordination of health care services $[1,2,4]$. Understanding how to create health care systems from the perspective of multimorbid patients aged more than 65 years may lead to improvement in health care services and consequently facilitate provision of better quality of life and reduction of functional disabilities among these individuals $[4,15]$. Since the existing literature perceives the concept of "continuity of care" differently, we consider it relevant to conduct a systematic review of the literature concerning older people over the age of 65 with multimorbidity and their experiences on integration of health care services. By using experiences on integration of health care services as a simple version of "continuity of care", we believe we are able to cover "continuity of care" and the aspects made in the different presumptions of "continuity of care". Therefore the objectives of the present review are to synthesize the manner in which older patients with multimorbidity experience the level of integration of health care services and to identify barriers to continuity of care.

\section{Methods}

We followed the PRISMA guidelines for systematic review in this review [16].

\section{Literature search}

The first author conducted a systematic literature search in February 2018 in the following databases: Scopus, Embase, CINAHL, and MEDLINE. The first author devised the search strategy in collaboration with a research librarian. The search string was adjusted to each database, using the MeSH, Emtree, or exact major subject heading (MM)/exact subject heading ( $\mathrm{MH})$ terms for each database. The following search strings were used:

(Comorbidity [mesh, emtree, MM] OR Multimorbidity [emtree] OR (Multimorbid" or multi-morbid* or comorbid* or co-morbid* or multidisease* or multi-disease* or frail" or vulnerab*) OR ((multipl* or cooccur*or co-exist*or co-occur*) adj3 (ill* or disease* or condition* or syndrom* or disorder* or symptom* or medication* or health*))) AND (Aged [mesh, emtree, $M M / M H]$ OR Aged, 80 and over [mesh, MM] OR Senescence [emtree] OR Very elderly [emtree] OR (old* or elder* or aged or geriatic* or senescence or senior* or senium or centenarian" or nonagenarian" or octogenarian* or genrontolog" or "late life")) AND (Patient Satisfaction [mesh, emtree, MM] OR Patient attitude [emtree, MM] OR Patient preference [emtree] OR ((Patient*) adj3 (Attitude* or opinion* understanding* or perspective* or satisfaction* or preference* or view* or standpoint* or perception* or experience*))) AND (Continuity of Patient Care[Mesh, MM/MH] OR Patient care [emtree] OR Delivery of Health Care, Integrated [mesh, MM] OR Integrated Health Care Systems [emtree] OR Intersectoral Collaboration [mesh, emtree] OR Cooperative Behavior [mesh] OR Cooperation [emtree] OR "contin" of patient care" OR "Patient Care Contin"” OR "Contin" of Care" OR "Care Contin"” OR "Coordinat" care" OR "multidisciplinary care" OR "multi-disciplinary care" OR "Intersectoral Collaboration" OR "Intersectoral Cooperation" "Integrated Delivery System" OR "Cooperative Behavior" OR "Compliant Behavior"”).

\section{Study selection}

After the literature search, the first author screened all studies twice using the titles and abstracts, with a gap of a week between each screening, using the systematic review management program Covidence [17]. Last author was consulted when in doubt. Next, both authors conducted full-text screenings separately. In case they disagreed on certain studies to be included in the full-text screening, first, these two authors discussed such studies and if they did not arrive at an agreement, then the other authors on the research team read and discussed the articles to arrive at a consensus.

\section{Inclusion criteria}

We included studies that fulfilled the following inclusion criteria:

- The studies had to explore the patients' viewpoints and address aspects such as their experience, and opinions about transitions, their relationship with their health care providers and relatives, the transition of information, and consistency of care and personnel and the health care system they were navigating within. The study participants had to be patients aged 65 years or older.

- The study participants had to be patients with multimorbidity. If this fact was not explicitly stated, only studies with participants aged 85 years or older were included owing to the high proportion of multimorbidity in this age group [4].

We excluded studies that were:

- not written in English, Danish, Norwegian, or Swedish - not peer-reviewed, published articles. 


\section{Data extraction Analysis}

We included only one quantitative study and therefore we chose to code and analyzed it integrated with the other studies, which were qualitative. Therefore, all results are presented narratively. We analyzed all studies using thematic synthesis [18]. The synthesis consisted of three stages. In the first stage, we coded each article line by line according to its meaning and content about the manner in which older patients with multimorbidity experienced transitions, their relationship with their health care providers and relatives, the transition of information, and consistency of care and personnel, creating several initial codes. In the second stage, we formed descriptive themes to capture the meaning of the initial codes. In the first and second stages, we stayed close to the original findings in the included studies. Creating the descriptive themes offered us the possibility to go beyond the content in the original studies. In stage three, we created analytical themes, which means we used the descriptive themes to find answers to our research questions [18]. We used QSR International's NVivo11 qualitative data analysis software to conduct the synthesis [19].

\section{Quality appraisal}

We used the CoreQ checklist for assessment of study quality [20]. In rating the quality of the included study, we focused only on domain 2, Study design (items 9, 10, $11,12,14,15,16$, and 17), and domain 3, Analysis and findings (items 26, 29, and 30). Each study was assigned one point if the items were identified; therefore, a study could obtain 0-11 points, with 11 being the highest score, thus indicating highest quality. We rated the quality high if the score was 8 to 11 , medium if 4 to 7 and low if 0 to 3 . We only included articles scoring 4 or higher. The first author conducted the quality assessment, and last author reviewed the results.

\section{Results}

\section{Literature search}

We identified a total of 2030 studies, 310 from Embase, 1282 from MEDLINE, 258 from Scopus, and 180 from CINAHL. An additional 15 records were added after identification by backward citation. After removal of 352 duplicates, 1693 studies remained for title and abstract screening. After title and abstract screening, we excluded 1618 studies and found 75 studies eligible for full-text screening. We excluded most studies because they did not meet the age criterion and the patient's viewpoint criterion. After the full-text screening, nine articles remained for quality assessment and final synthesis, as shown in the PRISMA Chart in Fig. 1. All these studies are on Western countries and are published between 2008 and 2017.They represent a broad variety of patient groups. The included papers focus on the patients experience with a transition between primary and secondary care setting or vice versa or on how the patients experience the health care system in general. Most interviews were conducted after the patient was discharged or had visited their general practitioner. Characteristics of the selected studies [21-29] are presented in Table 1.

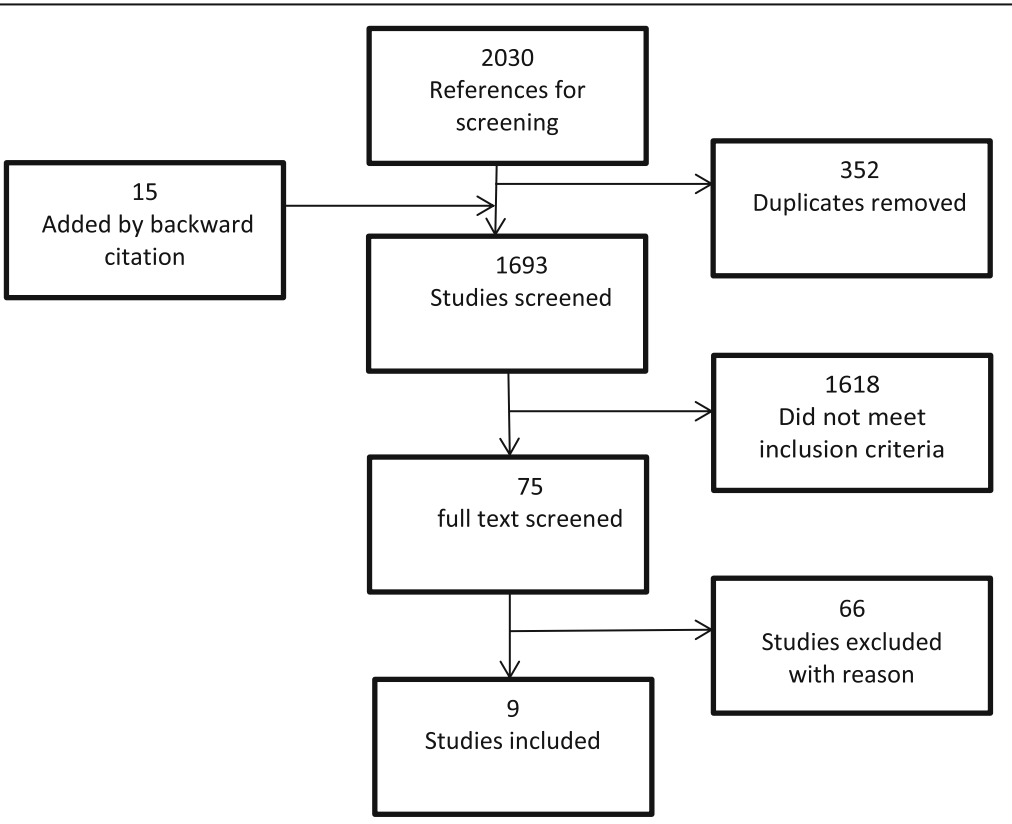

Fig. 1 PRISMA flow diagram 


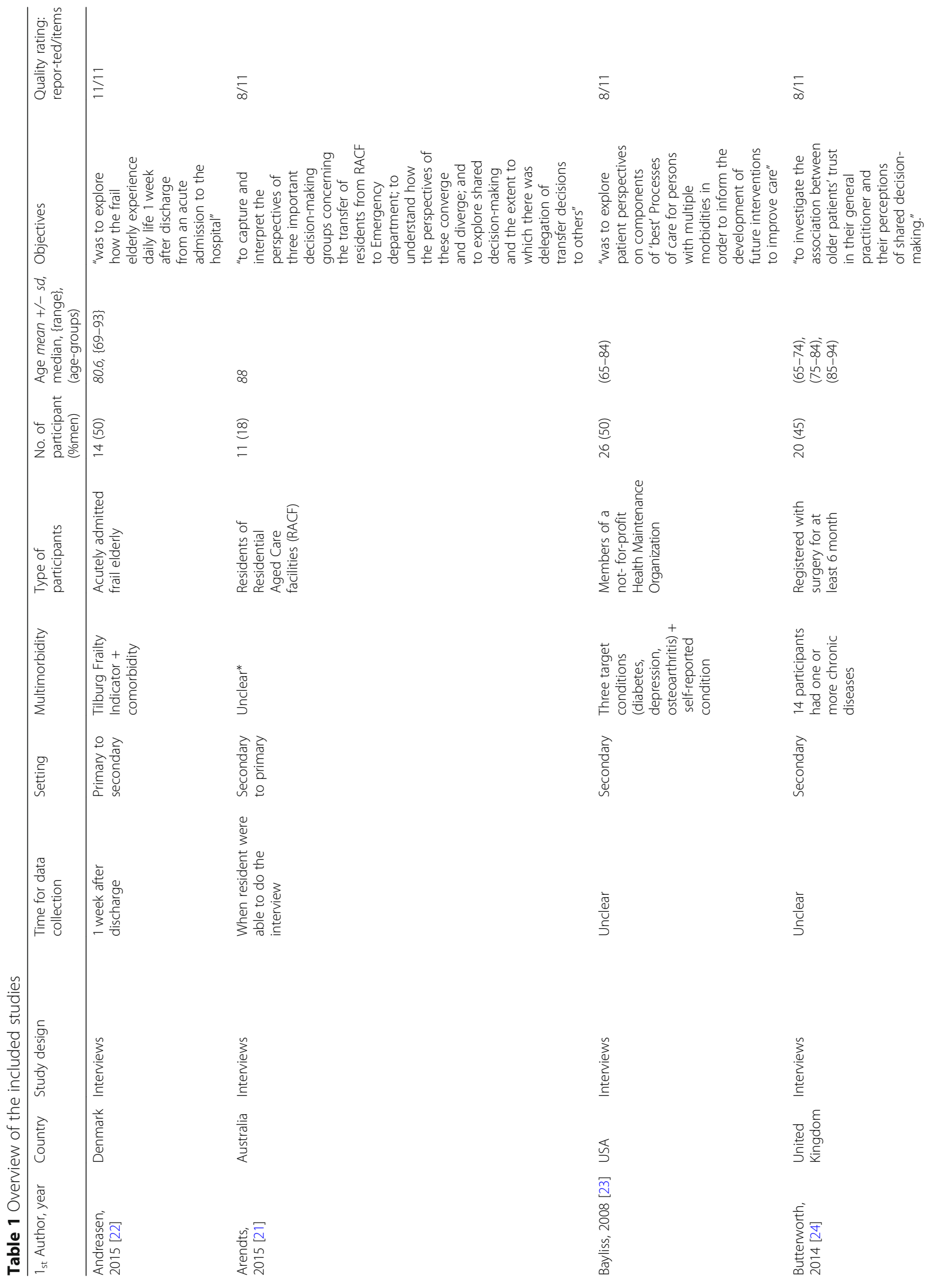




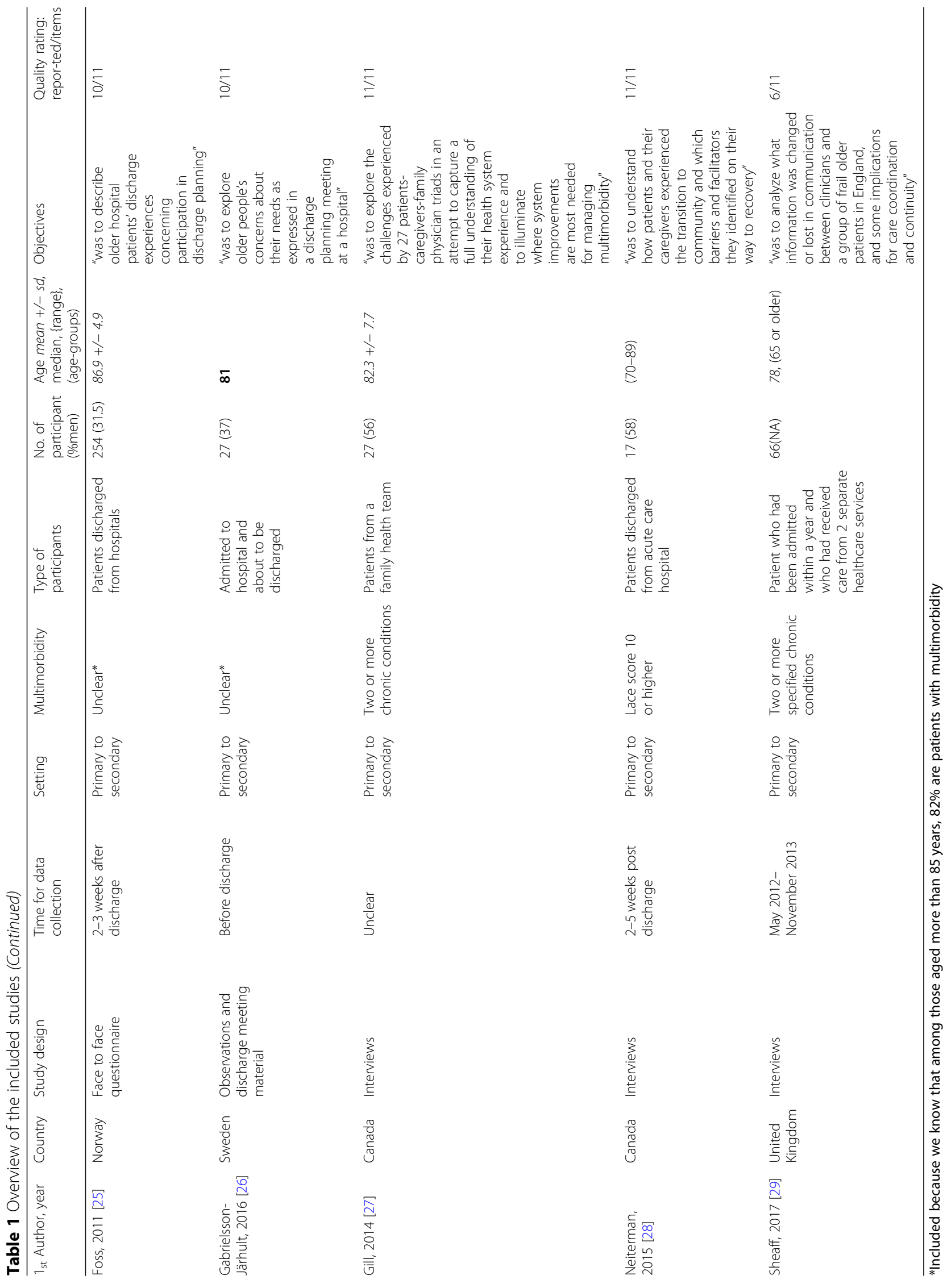




\section{Quality assessment}

We included all nine selected articles in the final synthesis, and rated these from medium to high quality. The quality rating is shown in Table 1 .

\section{Thematic synthesis}

The line-by-line coding resulted in 16 initial codes. In creating the descriptive themes, we searched all initial codes for similarities and differences and 10 descriptive themes emerged from this process. All 10 themes were centered on the experiences of the older multimorbid patients, and all contributed to three analytical themes. The three main issues that emerged from the studies on ways in which the older multimorbid patients experienced integrations of health care services were: (1) "Involved in the decisionmaking," (2) "Thoughts about transition," and (3) "Relationship to health care providers." The main themes overlapped each other, although only one [26] of the included studies contributed to all themes. The overlapping issues in the main themes are communication between the health care providers and the older multimorbid patient and granting individual needs of these patients. The coding tree is shown in Fig. 2.

\section{Continuity of care}

\section{Involved in decision making}

We found through the synthesis that the older multimorbid patients felt that the level of integration of services and coordination between health care providers was a success when they experienced being involved in the decision process and they felt listened to, and acknowledged for their awareness, about their own needs $[23,24,26]$. The patients who had been involved in the decisions process felt more trust in their health care provider [24]. When the multimorbid patients expressed that they had not being involved in the decision process regarding their own care [25], integrations of services were unsuccessful and it made them feel less trust towards their health care providers [21].

Thoughts about transition This theme captured that the older multimorbid patients experienced the level of integrations of services as successful when they had relatives to rely on in a transition [28] and when they felt a sense of security on transfer from an aged-care facility to an emergency department, because they felt safer at the latter [21]. When the integration of services and the coordination between health care providers did not succeed, these patients felt that they were discharged too early, and that made them feel insecure $[22,28]$. This finding also applied in situations in which they were discharged with no help from anyone other than their relatives [22]. When at home, these patients also experienced the feeling of being treated as an object by their health care providers [22] and that they did not receive the help they needed or were receiving help but not to the extent they perceived to be adequate [22, 26]. In particular, patients without relatives expressed their inability to assume responsibility for their own care [28]. They wanted to be listened to about their wishes for help in their own home [22]; experiences of disorganized post-discharge care were not uncommon [28].

Relationship to health care providers These older multimorbid patients experienced integrations of services as successful when they had convenient access to their health care providers [23] but felt frustration at times, especially when they had to wait for long periods [23, 27, 29] and when physicians only examined one health issue per visit [29]. Concurrently, the patients did not want to bother their physicians unduly [20,22]. The

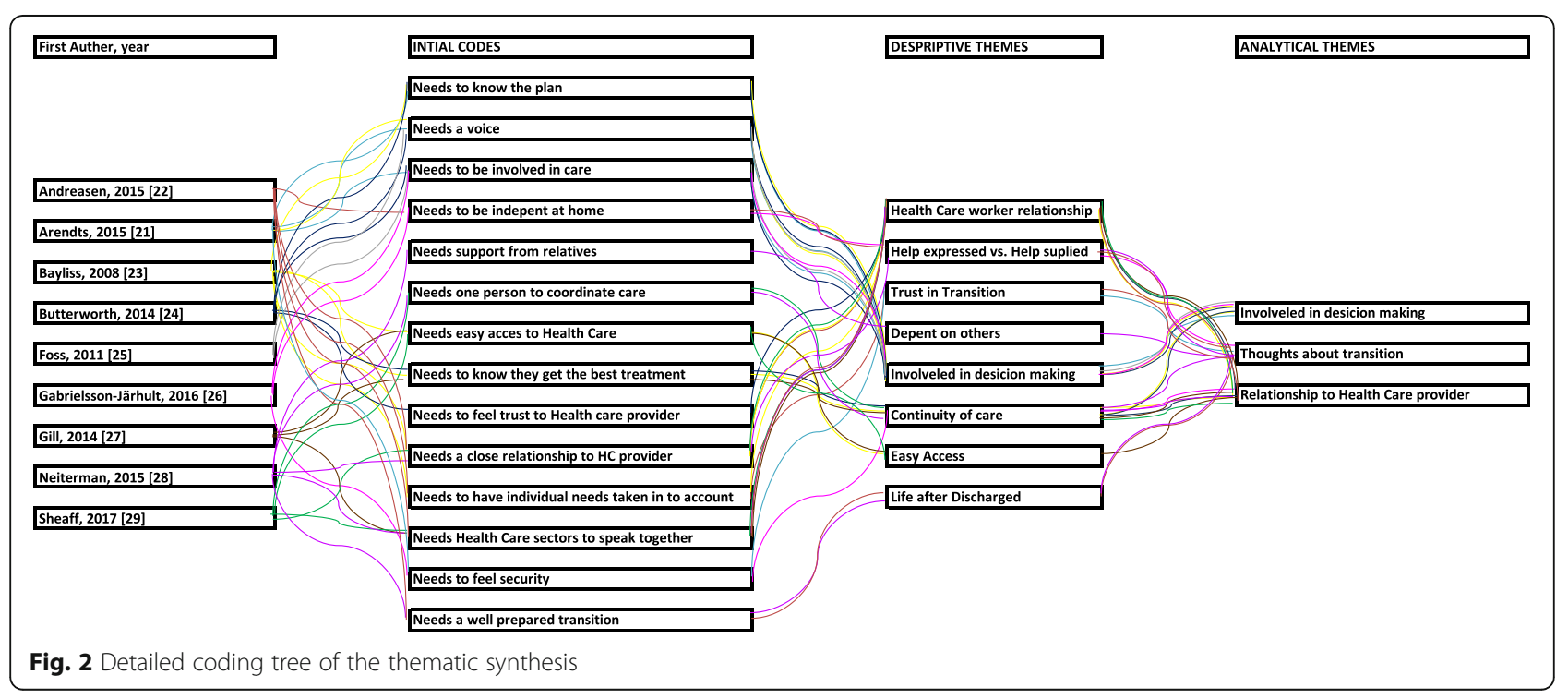


older multimorbid patients also needed to know that their health care provider as well as their general practitioner acted in patients' best interest [24].

Integration of services was also successful when the patients had a care coordinator to help them prioritize the demands that their multiple conditions imposed upon them and when the coordinator helped them keep track appointments and their health conditions [23, 28, 29]. The care coordinator could be the GP, and it was important for the patient to visit the same GP to obtain continuity $[24,29]$. These patients also perceived continuity of care when they felt listened to and the care was finalized from their point of view [24]. When the integration of services and the coordination between health care providers did not succeed, it was a barrier to obtaining continuity of care.

\section{Discussion}

\section{Principal findings}

This review shows that multimorbid patients (aged 65 years or older) in various settings experience both successful and unsuccessful integration of health care services. It was successful when the patients felt listened to on all the aspects of being individuals with multimorbidity and when they obtained help from a care coordinator to prioritize their appointments. However, they felt frustrated when they did not have easy access to their health providers, when they were not listened to, and when they felt they were discharged too early. These findings underpin the need for health care systems to adapt to the growing older population and people with multimorbidity and to find solutions on ways to create flexible systems that are able to help older patients with multimorbidity, meet their individual needs and their desire to be involved in decisions regarding their care.

\section{Continuity of care}

The thematic synthesis found three main themes, "Involved in decision making," "Thoughts about transition," and "Relationship to health care providers"; the overlapping issues were communication and granting individual needs for the older multimorbid patients.

From the themes "Involved in decision-making" and "Thoughts about transition," we know that these patients did not feel listened to and that they were not always participating in their discharge plan. This finding is not new knowledge [12], but it indicates that the health care systems still need improving and must focus on creating a better communication flow for older multimorbid patients. Studies have also found that involving patients in their discharge solutions and providing tailored individual plans may result in a small reduction in the length of hospital stay and reduce the probability of readmission rate within the 3 month [30]. Further, these patients have more trust in their health care provider when they are involved in the decision process. This finding is very interesting because it indicates that the more the patients' involvement, the more their trust in the health care system. We know that patients experience continuity when they feel trust in their health care provider and distrust when they experience gaps in their care [31-34], and it raises the following question: Does trust via patient involvement create a high level of integration of services and thereby a high level of "continuity of care"? The findings in this review indicate that it does.

Multimorbid patients have a high use of health care services [6], and as we know from the theme "Relationship to health care providers," it can be difficult for them to have easy access to their health care providers. Further, from "Thoughts about transition," we know that older multimorbid patients also request that their individual needs be considered when receiving home care from the health care system and that they do not always fit into the "standard" care. Certain studies have stated that prioritizing the demands that matter the most to these patients, by listening to them, is key to providing them the most appropriate care [35]. This finding indicates a need for flexible solutions. The need to create flexible solutions is one finding in the theme "Relationship to health care provider," in which we described these patients' desire to have a care coordinator to take care of their complex situation. Health care systems may be able to create a high level of continuity of care, if the older multimorbid patient has one person who is able to coordinate their care [36]. Studies have also stated that having a care coordinator is most important when a patient is multimorbid [31]. Being older individuals with multimorbidity places these individuals in a position where they may have limited resources [37]. Being an individual with multimorbidity is a complex situation, as findings from the three themes show, and as found in several other studies [3-6]. This fact raises the following question: How should the health care systems be changed to create a high level of integration of services and avoid the experiences that are a barrier to continuity of care?

\section{Strengths and weaknesses of the study}

The strength of this review lies not only in the systematic approach we used to find the included articles but also in the synthesis of the experiences of the integration of health care services from the older $(\geq 65)$ multimorbid patients' perspectives. We used thematic synthesis to address the manner in which these patients experience the level of integration of health care services and to identify barriers to continuity of care. Certain researchers may argue that it is not possible to synthesize qualitative research when the individual studies are decontextualized and the themes identified in one setting might not be applicable to other 
settings [18, 38].However we, as well as other researchers [18], believe that by checking that we did not interpret themes into settings to which they did not belong, we grounded the text in the context in which it was constructed. Other strengths are that this review contributes to knowledge in this area from the patients' perspectives, and all the studies included in this review are of medium to high quality based on design, analysis, and findings.

A weakness in this review is that it is a challenge performing a search on both individuals' "experiences" and "multimorbidity." The term multimorbidity is used in various ways, as is the term continuity of care. Therefore, we structured the search string to catch this variety. We believe we covered as much of the literature as possible by including the most-used terms for both multimorbidity and continuity of care. We acknowledge that we found 15 studies by backward citation, but none of them was included in the final review. The included studies differ in characteristics, which can be viewed as a limitation. However, we do believe that by including studies conducted across different settings, we can achieve a higher level of abstraction [38]. Another limitation is that only one author conducted the screening of the title and abstracts, which we mitigated by blind screening of the title and abstracts twice, 1 week apart.

\section{Conclusions}

This review adds that it is necessary to conduct highquality research on methods of considering the individual needs of multimorbid patients above the age of 65 years in developing health care systems. This review found consistent evidence that these older patients wish to have their individual needs taken into consideration in their care plan and they want to be listened to on all aspects of their care. Thereby, they also felt higher trust towards their health care providers. It may also be essential for these patients to have a care coordinator, and health care systems as well as the patients will benefit from having such coordinators. Appointing coordinators may even prevent these patients' unsuccessful experiences of not being involved in the decision process, their feelings of being discharged too early and of not receiving sufficient help after discharge, and their lack of easy access to health care providers. Thus, it would provide multimorbid patients the feeling of being listened to and having their individual needs considered and remove the barriers to achieving continuity of care. Potential research directions are to determine ways to integrate the older multimorbid patients' individual needs into a trustworthy health care system and to create flexible solutions in a standard system that includes differences and more vulnerable older patients without a strong social network and support.

\section{Abbreviations}

$\mathrm{MH}$ : Exact subject heading, search term in Cinahl database; MM: Exact major subject heading, search term in Cinahl database; RACF: Residential Aged Care facilities

\section{Acknowledgements}

The authors would like to thank Research Liberian Herdis Foverskov, University Library of Southern Denmark, for her expertise and collaboration in creating the search-string for this systematic review. We also like to thank OPEN, Odense Patient data Explorative Network, Odense University Hospital, Odense, Denmark for proving the research programs used in the article. We also like to thank the reviewers who provided relevant and constructive comments.

\section{Authors' contributions}

The first author conducted the literature search and conducted the screening in collaborations with the last author. The first author wrote the draft of the article. All authors contributed to the design, the revision and methodological considerations of the article. All authors have read and approved the final manuscript.

\section{Funding}

The project is funded by the Region of Southern Denmark, University Hospital of Southern Jutland, Danish municipalities': Aabenraa, Haderslev, Soenderborg, Toender and AP Moller Fonden. The funders had no role in the design of the study, in the collection of data, in the analysis, in the interpretation of data and in writing of the manuscript.

\section{Availability of data and materials}

The dataset used and analyzed during the current study, including a list of the excluded articles, are available from the corresponding author on reasonable request.

Ethics approval and consent to participate

Not applicable.

Consent for publication

Not applicable.

\section{Competing interests}

The authors declare that they have no competing interests.

\section{Author details}

${ }^{1}$ University Hospital of Southern Jutland, Kresten Philipsens vej 15, indgang F, 6200 Aabenraa, Denmark. ${ }^{2}$ Focused Research Unit of Emergency Medicine, Department of Regional Health Research, University of Southern Denmark, Odense, Denmark. ${ }^{3}$ Emergency Department, University Hospital of Southern Jutland, Jutland, Denmark. ${ }^{4}$ Kong Christian X's Gigthospital, Toldbodgade 3, 6300 Gråsten, Denmark. ${ }^{5}$ Focused Research Unit in Rheumatic, Department of Regional Health Research, University of Southern Denmark, Odense, Denmark. ${ }^{6}$ Research Unit of General Practice, Department of Public Health, University of Southern Denmark, J.B. Winsløws Vej 9, 5000 Odense, Denmark. ${ }^{7}$ Research Unit of Health Promotion, Department of Public Health, University of Southern Denmark, Niels Bohrs Vej 9-10, 6700 Esbjerg, Denmark.

Received: 27 June 2019 Accepted: 16 October 2019

Published online: 05 November 2019

\section{References}

1. World Health Organization. World Report on Ageing and Health. World Health Organization (online). Available at: http://apps.who.int/iris/ bitstream/10665/186463/1/9789240694811_eng.pdf.

2. Christensen K, Doblhammer G, Rau R, Vaupel JW. Ageing populations: the challenges ahead. Lancet. 2009;374:1196-208.

3. Salive ME. Multimorbidity in older adults. Epidemiol Rev. 2013;35:75-83.

4. Barnett K, Mercer SW, Norbury M, Watt G, Wyke S, Guthrie B. Epidemiology of multimorbidity and implications for health care, research, and medical education: a cross-sectional study. Lancet. 2012;380:37-43.

5. Marengoni A, Angleman S, Melis R, et al. Aging with multimorbidity: a systematic review of the literature. Ageing Res Rev. 2011;10:430-9. 
6. Roughead EE, Vitry Al, Caughey GE, Gilbert AL. Multimorbidity, care complexity and prescribing for the elderly. Aging Health. 2011;7:695-705.

7. Tinetti ME, Fried TR, Boyd CM. Designing health care for the most common chronic condition - multimorbidity. JAMA. 2012;307:2493-4.

8. Schoen C, Osborn R, How SKH, Doty MM, Peugh J. In chronic condition: experiences of patients with complex health care needs, in eight countries, 2008. Health Aff. 2009;28:w1-16.

9. Haggerty $U$, Reid RJ, Freeman GK, Starfield BH, Adair CE, McKendry R. Continuity of care: a multidisciplinary review. BMJ. 2003;327:1219-21.

10. Freeman G, Sheppard S, Robinson I, Ehrich K, Richards S. Continuity of care: report of a scoping exercise for the National co-ordinating Centre for NHS service delivery and Organisation R \& D (NCCSDO), 2000.

11. Reid R, Haggerty J, McKendry R. Defusing the confusion: concepts and measures of continuity of healthcare. Canadian Health Services Research Foundation, 2002.

12. Cornwell J, Levenson R, Sonola L, Poteliakhoff E. Continuity of care for older hospital patients : a call for action Eds. London: The King's Fund; 2012.

13. MacAdam M. Frameworks of integrated care for the elderly: a systematic review. Canadian Policy Research Networks Research report, 2008.

14. Guthrie B, Saultz JW, Freeman GK, Haggerty JL. Continuity of care matters. BMJ (online). 2008;337.

15. Boyd CM, Fortin M. Future of multimorbidity research: How should understanding of multimorbidity inform health system design? Public Health Rev. 2010;32:451-74.

16. Moher D, Liberati A, Tetzlaff J, Altman DG. The PRISMA group Preferred reporting items for systematic reviews and meta-analyses: The PRISMA Statement. PLoS Med. 2009;6:e1000097.

17. Covidence systematic review software. Veritas Health Innovation, Melbourne, Australia.

18. Thomas J, Harden A. Methods for the thematic synthesis of qualitative research in systematic reviews. BMC Med Res Methodol. 2008:8:45.

19. QSR International Pty Ltd. NVivo qualitative data analysis software. Version. 2012;11.

20. Tong A. Consolidated criteria for reporting qualitative research (COREQ): a 32-item checklist for interviews and focus groups. Int J Qual Health Care. 2007:19:349-57.

21. Arendts G, Popescu A, Howting D, Quine S, Howard K. 'They never talked to me about... ': Perspectives on aged care resident transfer to emergency departments. Australas J Ageing. 2015;34:95-102.

22. Andreasen J, Lund $H$, Aadahl M, Sorensen EE. The experience of daily life of acutely admitted frail elderly patients one week after discharge from the hospital. Int J Qual Stud Health Well-being. 2015;10:27370.

23. Bayliss EA, Edwards AE, Steiner JF, Main DS. Processes of care desired by elderly patients with multimorbidities. Fam Pract. 2008;25:287-93.

24. Butterworth JE, Campbell JL. Older patients and their GPS: shared decision making in enhancing trust. Br J Gen Pract. 2014;64:e709-18.

25. Foss C, Hofoss D. Elderly persons' experiences of participation in hospital discharge process. Patient Educ Couns. 2011:85:68-73.

26. Gabrielsson-Järhult F, Nilsen P. On the threshold: older people's concerns about needs after discharge from hospital. Scand J Caring Sci. 2016;30:135-44.

27. Gill A, Kuluski K, Jaakkimainen L, Naganathan G, Upshur R, Wodchis WP. "where do we go from here?" health system frustrations expressed by patients with multimorbidity, their caregivers and family physicians. Healthc Policy. 2014;9:73-89.

28. Neiterman E, Wodchis WP, Bourgeault IL. Experiences of older adults in transition from hospital to community. Can J Aging. 2015;34:90-9.

29. Sheaff $R$, Halliday J, Byng $R$, et al. Bridging the discursive gap between lay and medical discourse in care coordination. Sociol Health IIIn. 2017;39:1019-34.

30. Gonçalves-Bradley DC, Lannin NA, Clemson LM, Cameron ID, Shepperd S. Discharge planning from hospital. Cochrane Database of Systematic Review. 2016;1.

31. Haggerty JL. Ordering the chaos for patients with multimorbidity. BMJ. 2012;345:e5915.

32. von Bültzingslöwen I. Patients' views on interpersonal continuity in primary care: a sense of security based on four core foundations. Fam Pract. 2006;23:210-9.

33. Waibel S, Henao D, Aller MB, Vargas I, Vazquez ML. What do we know about patients' perceptions of continuity of care? A meta-synthesis of qualitative studies. Int J Qual Health Care. 2012;24:39-48.

34. Haggerty JL. Experienced continuity of care when patients see multiple clinicians: a qualitative metasummary. Ann Fam Med. 2013;11:262-71.
35. Roland M, Paddison C. Better management of patients with multimorbidity. BMJ. 2013;346:f2510.

36. Peikes D. Effects of care coordination on hospitalization, quality of care, and health care expenditures among medicare beneficiaries: 15 randomized trials. JAMA. 2009;301(6):603-18.

37. Sudore RL. Limited literacy in older people and disparities in health and healthcare access. J Am Geriatr Soc. 2006;54:770-6.

38. Britten N, Campbell R, Pope C, Donovan J, Morgan M, Pill R. Using meta ethnography to synthesise qualitative research: a worked example. J Health Serv Res Policy. 2002;7:209-15.

\section{Publisher's Note}

Springer Nature remains neutral with regard to jurisdictional claims in published maps and institutional affiliations.
Ready to submit your research? Choose BMC and benefit from:

- fast, convenient online submission

- thorough peer review by experienced researchers in your field

- rapid publication on acceptance

- support for research data, including large and complex data types

- gold Open Access which fosters wider collaboration and increased citations

- maximum visibility for your research: over $100 \mathrm{M}$ website views per year

At BMC, research is always in progress.

Learn more biomedcentral.com/submissions 